
maps. In S. Hirtle \& A. Frank (Eds.), Spatial information theory: A theoretical basis for GIS (pp. 347-361). Berlin: Springer.

\title{
Cognitive Requirements on Making and Interpreting Maps ${ }^{1}$
}

\author{
Thomas Barkowsky and Christian Freksa \\ Department of Computer Science and \\ Doctoral Program in Cognitive Science \\ University of Hamburg \\ Vogt-Kölln-Str. 30 \\ D - 22527 Hamburg, Germany \\ \{barkowsky, freksa\}@informatik.uni-hamburg.de
}

\begin{abstract}
We present an approach to modeling human interpretation of (real) geographic maps. While in Geographic Information Systems (GIS) the limitations for describing geographic knowledge mainly stem from the limitations of this knowledge itself, paper maps pose additional constraints on the representation of spatial configurations. We examine maps as representation media with respect to cartographic restrictions involved in the map making process. Some cognitive factors of cartographic generalization are indicated. We present our aspect map approach allowing for describing maps formally as pictorial representations. The approach postulates the use of meta-knowledge to enable adequate map interpretation. Phenomena of cartographic interpretation and misinterpretation are illustrated employing two kinds of hierarchic structures of spatial aspects of maps. The notions we present can be employed in augmenting the 'cognitive adequacy' of automated map making and map reading.
\end{abstract}

\section{Introduction}

Maps as representational media have proven useful for dealing with geographic knowledge over centuries [Bagrow 1985]; they have become a natural means for accessing and processing this knowledge in our culture. But although cartography as a scientific area of competence has been improving over a long time, it still lacks a formal foundation for many of the aspects it is confronted with (cf. [MacEachren 1995, Head 1991]). Over the last four decades or so, cartography has been facing major changes in at least three ways: (1) together with early computer technology, the map making process has been supported by facilities of collecting and maintaining data and by supporting map design by means of computer graphics and computer aided

\footnotetext{
1 This work is supported by the Deutsche Forschungsgemeinschaft (DFG) in the framework of the priority program on spatial cognition (grant Fr 806/8).
} 
design techniques; (2) in the consequence of the former, Geographic Information Systems (GIS) have been developed to integrate the processes of data acquisition, manipulation, and presentation; and finally, (3) with the approaches of cognitive science, the process of understanding maps is being explored to gain insight in the ways humans perceive and construct the surrounding geographic world through maps.

This paper is concerned with the third of the three aspects. Its endeavor lies in contributing to explain how people employ the medium 'map' within a representational system. This representational system conveys knowledge about environmental and geographic spaces by means of depicting objects in pictorial space ${ }^{2}$ [Montello 1993].

\section{Maps as Cognitive Interfaces to the Geographic World}

\subsection{Human-made Maps vs. Geographic Information Systems}

Map-making is an art which is not yet fully understood in formal terms. Map-makers are faced with difficult design decisions, as the medium paper does not meet all requirements of ideal maps simultaneously. Consequently, some constraints must be relaxed at the benefit of others. This can be done globally (i.e., the same constraints are relaxed in a certain way in the entire map) or it can be done locally (i.e. in each conflict situation the designer decides locally which constraint should be relaxed). In computer-generated maps usually the first approach is taken, as it is more systematic and easier to formalize. The second approach, however, can take into account the expected use of the map and can attempt to maximize the benefit to the user in each individual situation.

A GIS first of all consists of geographic data which describes geographic reality according to a conceptualization on a certain level of granularity. This data is intended to be processed by the system to yield formally correct results. The presentation of the stored or newly computed data on an output device - though essential for the practical use of the system - is usually of secondary significance. Although there is a strong interest in automating the complete map making process from data acquisition to map generation, producing maps of the quality known from human expert map makers is still an unsolved task in computer cartography [Frank \& Timpf 1994].

Both, databases in GIS and paper maps are the result of human conceptualizations of geographic space. While the main strength of GIS is the preservation of correct knowledge, the main strength of good paper maps is the communication of relevant knowledge. In paper maps, the underlying data is no longer directly accessible as in GIS. In the map making process the data effectively needs to be modified to meet the requirements of the communication process [Bertin 1981]. Human-made maps are

\footnotetext{
${ }^{2}$ We are predominately concerned here with visual maps; however, for other kinds of maps (e.g. tactile maps for the blind) the considerations presented in this paper may be applicable as well.
} 
inherently visual media which as external representations depend upon strong spatial constraints. These constraints are constitutive for the mapping as well as for the map reading process (see next chapter).

So, why is it sensible to examine interpretation processes of human-made geographic maps? One of the main claims of Naive Geography [Egenhofer \& Mark 1995 ] is that maps provide a very natural means to explore geographic space and that people perceive map space as more real then the experienced actual geographic environment itself. Regarding human map reading this leads to the insight that maps are more than a medium for depicting and communicating already existing spatial knowledge. Maps constitute spatial knowledge by providing a form to construct geographic reality (cf. [Head 1991]). This view on geographic maps suggests that the examination of human interaction with maps should be a fundamental approach to understanding spatial cognition in large scale spaces.

For these reasons, we develop here an approach to model the way maps are interpreted by map readers with respect to their spatial content. The objective of the present work is to model the relation between the map as representational medium and the spatial knowledge encoded therein.

The insights gained should enhance our understanding of pictorial/symbolic interaction and help improve the communication power of Geographic Information Systems.

\subsection{Maps as Integrated Spatio-Symbolic Media}

The conflict between constraints imposed on maps mainly stems from the fact that maps are neither scaled down copies of the surface of the depicted environment (spatial relation preserving geometric miniatures) nor are they geometric projections of the environment (like photographs). Maps integrate geometric projection with symbolic representation of processed information [Barkowsky et al., in press]. Both, geometric image and symbols compete for the same space in the representation medium and both types of information are relevant for the map user: without symbolic information, the user does not know what is depicted in the map and without geometry, the user does not know where the depicted items are located, what shape they have, and what their spatial relationships are.

A map can be viewed as a geometric projection in which the projected entities are replaced by symbolic interpretations of these entities. To maintain geometric fidelity, the transformed entities should keep the precise position, size and shape of the original projections. On a spatially 2-dimensional medium, this would only leave color to code the symbolic dimensions, which would make maps very difficult to read. Thus, to provide a wider channel for conveying symbolic content, we must give up the constraint of strict geometric correctness. Then we can employ the spatial dimensions partially to convey symbolic aspects in the map. 


\section{Cartography as a Representation Task}

\subsection{Representational Aspects of Paper Maps}

Maps are representations of the geographic world. Though this appears quite obvious, some problems occur when regarding maps as representations. Maps are constructed on the basis of some underlying set of geographic data. This data may, for example, be conceptualized in terms of a geographic database or it may be available in form of a topographic map of sufficiently high resolution. The act of map-making consists of a transformation $\mathrm{C}$ of the geographic conceptualization to the map to be generated. Ideally, we would like to interpret the map by inverting the transformation $\mathrm{C}$ to obtain the original geographic information encoded in the map (see Fig. 1).

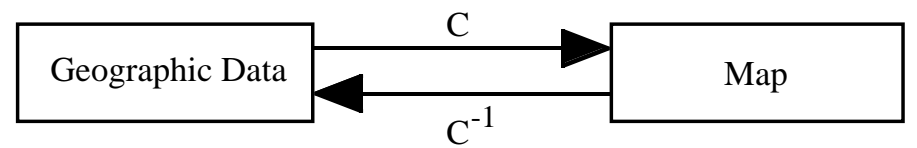

Fig. 1. Ideal correspondence between mapping (C) and interpretation $\left(\mathrm{C}^{-1}\right)$ processes

However, when confronted with paper maps, the process of generating maps can not easily be reversed for two reasons.

First of all - and trivially - generating a map involves loss of information, in general. For example, when an overview map of a spatial area is to be constructed from a given detailed reference map, reduction of information is required to preserve the readability of the new map. The phenomenon of information reduction involved in transforming one cartographic representation to another is known as cartographic generalization.

The second aspect is more essential. Loss of information due to cartographic generalization would be tractable if the processes of map-making were formally described and known by the map interpreter. But for two reasons this assumption does not hold in practice. On the one hand, a map typically is not provided with the information of how it has been generated from the underlying data ${ }^{3}$. On the other hand, the process of making maps cannot be fully described formally as will be illustrated in section 3.3.

\footnotetext{
3 The legend provided with each reasonable map (implicitly or explicitly) does not fulfill this function completely. It only explains how the symbols in the map are associated with geographic objects conceived on the map's intended level of granularity; however, it does not, tell about the act of generating the cartographic entities from the original geographic data.
} 
So far, we can state that maps as representational systems do not fulfill all requirements of conventional knowledge representation theory [Palmer 1978]. The notion of a representation system comprises full knowledge about the correspondence between the aspects to be represented (e.g. relations between geographic objects) and the components of the representing world (i.e. the cartographic entities). Before elaborating how these specific representational characteristics of maps can be treated more formally, we will exhibit some properties of cartographic generalization.

\subsection{Cartographic Generalization}

Cartographic signs on maps are restricted by the minimum size of graphic reproducibility and of visual recognizability as a lower bound of resolution. Therefore, the generation of overview maps (i.e. maps of lower spatial resolution) from detailed topographic maps requires cartographic generalization. Furthermore, maps as communication media need to meet the cartographic principle of constant information density which restricts the design of cartographic representations (cf. [Frank \& Timpf 1994]).

In cartographic generalization, two ways of simplifying the readability of maps are combined [Hake \& Grünreich 1994] ${ }^{4}$ :

(1) To facilitate recognition of the entities in the map, their size often needs to be enlarged. This operation violates the cartographic principle of geometric correctness. Furthermore, enlarging objects may cause other objects in the map to be displaced. A familiar example for enlarging cartographic objects is the broadening of linear cartographic entities such as streets or rivers.

(2) To save space on the map, geographic objects may be simplified, grouped with other objects, or totally omitted if they are of lesser importance. These operations generally violate the cartographic principle of completeness. A well known example of simplification is the straightening of linear cartographic entities (e.g. coastlines) or the symbolization of a town by a filled circle of fixed size.

These operations of cartographic generalization lead to a classification of extended cartographic objects into four categories [Hake \& Grünreich 1994]:

(1) the shape of the cartographic entity is equal to that of the object it stands for;

(2) the shape of the cartographic entity is only similar to the represented geographic object;

(3) only the center points of both, the cartographic and the geographic object, correspond to each other w.r.t. their position; and

(4) only the rough form and / or the approximate position of geographic objects are represented by the cartographic entities.

\footnotetext{
4 Though it is well known that cartography highly depends on the cultural background of the cartographer as well as the map user (see e.g. [Monmonier 1996]) we restrict ourselves to German cartographic examples in this paper. However, the principles should be valid for other cartographic traditions as well and the variability of representational methods only accounts for the degrees of freedom in cartography.
} 
Clearly, the precision of the cartographic representation w.r.t. the world it represents decreases from (1) (e.g. in large scale topographic reference maps) to (4) (e.g. rough low resolution overview maps) where reliable deductions of local situations are hardly possible.

Taking into account the insight that formalizing the interpretation of maps w.r.t. a representation theory requires formal knowledge on the map making process, it becomes desirable to automate the process of cartographic generalization accordingly.

\subsection{Formalizing Cartographic Generalization}

Map design is still a process that highly depends on the expertise of human cartographers, for both the rules of graphic communication and the map's intended purpose have to be taken into account [Bertin 1981, Kosslyn 1994].

But even when there are established cartographic rules for dealing with conflict situations, it is difficult to express them in formal terms. Moreover, formal descriptions of generalization processes do not automatically lead to 'objective' maps: the map reader never will be released from the interpretation task. The main benefit of automatically generalized geographic maps is that the resulting map can be compared with other maps generated the same way [Hake \& Grünreich 1994]. Nevertheless, the search for formal methods of dealing with cartographic generalization is a central endeavor in modern cartography (cf. [Frank \& Timpf 1994]).

One main access to automating cartographic generalization, which is of interest for the scope of this contribution, is to develop a hierarchic order for the generalization of different classes of cartographic objects. For in a given kind of map (e.g. a road map) we expect some pieces of information to be superior to others w.r.t. their authenticity (e.g. regarding their positions or shapes). In a road map, for example, depicting cities and the roads connecting them is essential whereas their precise locations or shapes are of lesser interest and therefore can be more easily modified for generalization purposes. The knowledge about this cartographic practice can lead us to an estimate about the genuineness of an aspect (i.e. some information that can be inspected in the map) under consideration. In this context w.r.t. the interpretation process it has to be mentioned that cartographic generalization clearly does not only affect the depiction of the objects themselves, but also the relations between them. For example, two objects which are connected in the geographic world must not be separated in the map generalization process for reasons that refer to each of the objects individually.

In summary, automatic cartographic generalization mostly leads to spatial conflicts in the depiction of cartographic entities. These conflicts inevitably require to be visually detected and corrected by a human expert. A formal approach to modeling the interpretation of paper maps has to deal with two major difficulties: (1) the transformation of the underlying geographic data to the map under consideration involves loss of information, (2) cartographic generalization has not yet been fully formalized, and (3) the choices in the generalization process taken by the cartographer are not known to the map reader. 
Nevertheless, maps work. Even non-professional map readers more often find the answers they are looking for in maps than they fail. So people succeed in combining their general spatial knowledge with the contents of maps in such a way that an overall inference works even if the individual contributing pieces of knowledge appear deficient. A formal model of the cognitive process of map interpretation has to describe how relations between cartographic entities can be associated with relations between geographic objects on the intended level of granularity induced by the map at hand.

\section{Existing Approaches to Formalizing Map Knowledge}

\subsection{Maps and Semiotics}

In the last decade or so there have been several attempts to regard the representational medium 'map' from the viewpoint of semiotics (e.g. [Schlichtmann 1985, 1991; Wood \& Fels 1986; Head 1991]). Semiotics, as a theory of signs and signification, deals with human communication by means of organized signification systems [Eco 1976]. Accordingly, cartographic depictions can be seen as communication systems consisting of cartographic signs which are employed within a system of signification, the map.

In semiotic theory, a sign is regarded as consisting of two components [Head 1991]: (1) It has a physical extension which depends on the form of communication (e.g. a sound, a gesture, or a mark on a piece of paper). The physical extension is usually called the expression of the sign. And (2) every sign has a content, i.e. its meaning or mental concept it refers to. For the purpose of using signs within a signification system, their contents and their expressions have to be linked by a code that is common to the people involved in the communication process.

With respect to cartography, it is obvious that the two components of the semiotic term 'sign' are on the one hand the cartographic entities placed in the map and on the other hand the geographic knowledge that corresponds to them. As we have seen in the previous sections in regarding maps it is sensible to differentiate between those two components. For in the map-making as well as in the map reading process there are always two instances of knowledge involved: the map with its cartographic entities and the underlying mental concepts of the cartographer or the map reader.

\subsection{Formal Map Semantics}

The map semantics project [Pratt 1993] is an attempt to provide a formal semantics of cartographic representation in analogy to formal languages. The goal is to develop criteria to decide whether a given cartographic representation is true or false and under which conditions it might be true. To provide a formal semantics a major concern is to decide which entities on the map form the map's symbols and which belong to the 
map's background. This question is paralleled in the psychological figure-ground dichotomy [Kanizsa 1979]. With respect to maps, however, it becomes apparent that there is no unique figure-ground differentiation, for in every map there are many possibilities of organizing cartographic entities which may be meaningful in different situations. Therefore, it only depends on the map user's intention how the map is seen in a given context.

According to the map semantics approach a map (or rather a map-like representation) has two characteristics [Lemon \& Pratt 1996]: (1) maps are only approximately true, i.e. they have a certain verisimilitude w.r.t. an underlying geographic representation which is used as reference; (2) maps exhibit a number of typical errors which a theory of formal semantics has to account for. Among those errors are the depiction or non-depiction of non-existent or existent objects, respectively; the incorrect representation of shapes of geographic objects; or errors in location. These examples show that in the map semantics approach maps are idealized in two ways. Firstly, map-like representations are assumed to be complete w.r.t. the underlying reference - any incompleteness is regarded as erroneous. Secondly, it is assumed that maps are intended to be exact. Both idealizations can be regarded as contradictory w.r.t. the necessity of cartographic generalization (at least when midscale and small-scale maps are involved).

Deviations from idealized true map-like representations are therefore treated as imprecisions or errors, in the map semantics project. These deviations are formally described as imperfect information flow by means of a suitable form of channel theory [Lemon \& Pratt 1996]. An information channel is a set of interpretational conventions which leads to an approximation semantics providing a criterion for a measure of exactness for a given map.

\section{The Aspect Map Approach}

As we have seen by regarding cartographic generalization, maps are designed to present only certain pieces of knowledge about geographic entities. The fact, that a map selects pieces of knowledge or aspects leads us to the notion of aspect maps. An aspect map is a formal description of a map that allows for distinguishing between intended or representational pieces of information and those incorporated by the pictorial property of over-representation (cf. [Wang 1995]).

An aspect map is described by a triple $\langle E, R, M>$ wherein $E$ is a set of entities, $R$ is a set of relations that hold between these entities, and $M$ is a reference to the metaknowledge about the pictorial contents described by $\mathrm{E}$ and $\mathrm{R}$. We shall exhibit these components in more detail in the subsequent sections.

\subsection{Representing Aspect Maps}

Our approach assumes the availability of a set E of identified cartographic entities contained in the map and the appropriate processes yielding the spatial relations $\mathrm{R}$ 
between these entities. Thus, $\mathrm{R}$ contains the spatial relations that hold in the map; in particular, it contains the positions of the entities in the map, the extensions of linear entities or areas, and the spatial relations that hold between two or more entities in the map.

So far, the spatial representation is not yet interpreted as a medium for conveying geographic knowledge. As we have seen in chapter 3, what is required for understanding a map is some additional knowledge about the way the depicted aspects must be interpreted. Therefore, some link between the spatial relationships in the map as a depiction and the underlying spatial knowledge encoded therein is needed. In our approach, that link is provided by the meta-knowledge reference $\mathrm{M}$.

In this way we distinguish between the map as an uninterpreted pictorial medium (denoted by the set of entities $\mathrm{E}$ and the set of relations R) and the connection to the underlying spatial knowledge (provided by the link to the meta-knowledge $\mathrm{M}$ ); the meta-knowledge $\mathrm{M}$ turns the picture into a spatial representation according to the usual understanding of the term 'geographic map'.

\subsection{Meta-Knowledge}

By the link $\mathrm{M}$ to the meta-knowledge, the information depicted in the map is connected to formal descriptions of spatial knowledge (compare [Freksa \& Röhrig 1993]). So every relation $R$ that can be inspected in the map can be assigned a corresponding translation to a suitable spatial calculus. The meta-knowledge itself can be grasped as the complete body of knowledge that comprises every possible reference between depicted relations of cartographic entities and geographic relations between the objects represented by them. Therefore, every possible combination between purely projective and purely symbolic ways of cartographic depiction can be covered, thus accounting for the diverse ways of modifying entities in cartographic generalization (cf. section 3.2).

To illustrate this, let us consider a simple example demonstrating some extreme differences in the way the map contents may have to be treated. Fig. 2 shows a fragment of the Hamburg subway network map. It is a special kind of schematic survey map with a liberally relaxed geometric correspondence to its topographic basis. Three examples shall demonstrate how depicted aspects need to be taken in representationally different ways. (1) The depicted connections between stations can be taken as literally representing the connectedness of the stations under consideration. (2) The orientations of the stations w.r.t. each other - depicted by some precise angles - must not be taken as representing precise orientations, but they might be assigned some coarse orientation pattern, e.g. according to a qualitative 8-sector classification. (3) The distances between station symbols in the map do not reflect distances between the corresponding stations, thus forbidding even qualitative comparisons between them. 


\subsection{Interpretation and Misinterpretation}

Interpreting maps involves transformation of perceivable relationships of cartographic entities on the map to spatial information about the corresponding geographic objects. The correspondence between the entities in the map and the geographic objects is given in our model by the link to meta-knowledge. Therefore modeling a correct map interpretation using aspect maps means using the correct meta-knowledge for the map at hand.

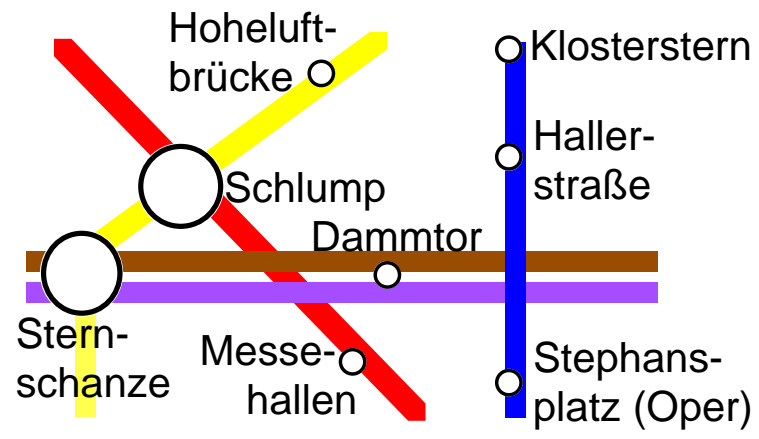

Fig. 2. Example of an aspect map: Hamburg subway network map (fragment).

In everyday use, maps often are misinterpreted; i.e. cartographic entities and the relations between them are incorrectly related to the geographic objects in the world. In terms of modeling map interpretation with aspect maps this means that a reference to the wrong meta-knowledge is used. The misinterpretation that presumably occurs most often is overinterpretation. That means that aspects depicted in the map are taken more literally than they are intended by the cartographer. In the above example (see Fig. 2) overinterpretation of the aspect of orientation could mean that the angles formed between subway station symbols in the map are taken as precise geometric orientations between the real subway stations.

\subsection{Hierarchies of Aspects}

The phenomenon of overinterpretation leads to the intuition that components of metaknowledge w.r.t. an aspect (e.g. orientation) can be arranged in a (partial) hierarchic order; i.e., the correspondences between the depiction in the map and the spatial relation in the geographic world can be ordered hierarchically by the strength of their representational correspondence. The aspect category of orientation, for example, may be arranged in a way as depicted in Fig. 3. With the arrangements of the various classes of meta-knowledge, the phenomenon of cartographic over- or underrepresentation is modeled by choosing a meta-knowledge reference at a wrong level of representational correspondence within this hierarchy. 
As we have motivated in section 2.2, in the process of map-making several classes of aspects compete with each other for specific map locations. Decisions about generalization steps are often taken locally by the cartographer by preferring one aspect over another. This leads to the effect that an aspect usually represented correctly in the map may be weakened in a particular situation in order to enable the correct depiction of a competing aspect which is regarded more important for the map's intended purpose. We already mentioned this phenomenon (see section 3.3) as an important issue in automating generalization processes by defining the order in which different aspect classes have to be generalized.
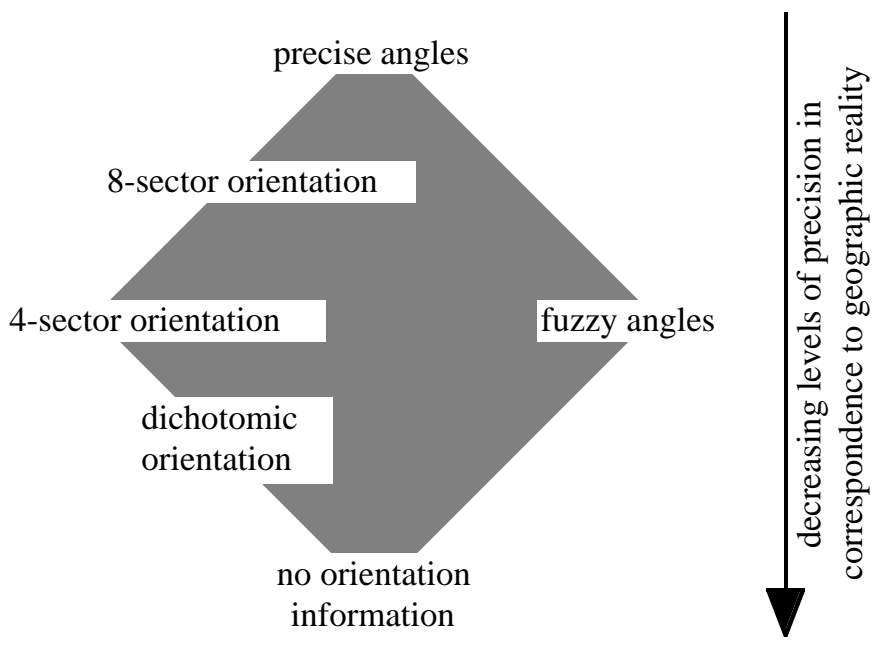

Fig. 3. Example of a hierarchic structure for the representation correspondence

It therefore seems sensible to impose a second hierarchic order of aspect depiction on a map for modeling interpretation processes: the depictional precedence between different classes of aspects (see Fig. 4). An aspect being ranked in a lower position within this hierarchy of aspect classes means that this aspect may be weakened if this is required for correctly representing some higher priority aspect.

With the use of the two described classes of hierarchies - the hierarchies of metaknowledge and those of depictional precedence - we can provide a model for map interpretation processes. Every map interpretation depends on some underlying assumptions on the kind of aspects being encoded in the map (as no map is provided with a formal description of its generation process). So for every kind of aspect to be interpreted in a given map (e.g. distance between locations) it is sensible to assume the existence of the map reader's assumption about the cartographer's intended level of precision. Reading a map using the correct level of meta-knowledge for any aspect to be interpreted usually leads to a correct interpretation result. 
Nevertheless, for reasons of different aspects competing for map locations, locally relaxed correctness may lead to inconsistent interpretation results, even when the correct level of meta-knowledge has been used. In this case, to deal with the unsatisfactory (incorrect) result, the depictional precedence hierarchy has to be used to relax the interpretation of those aspects whose correct depiction is assumed to be of lesser importance for map interpretation.

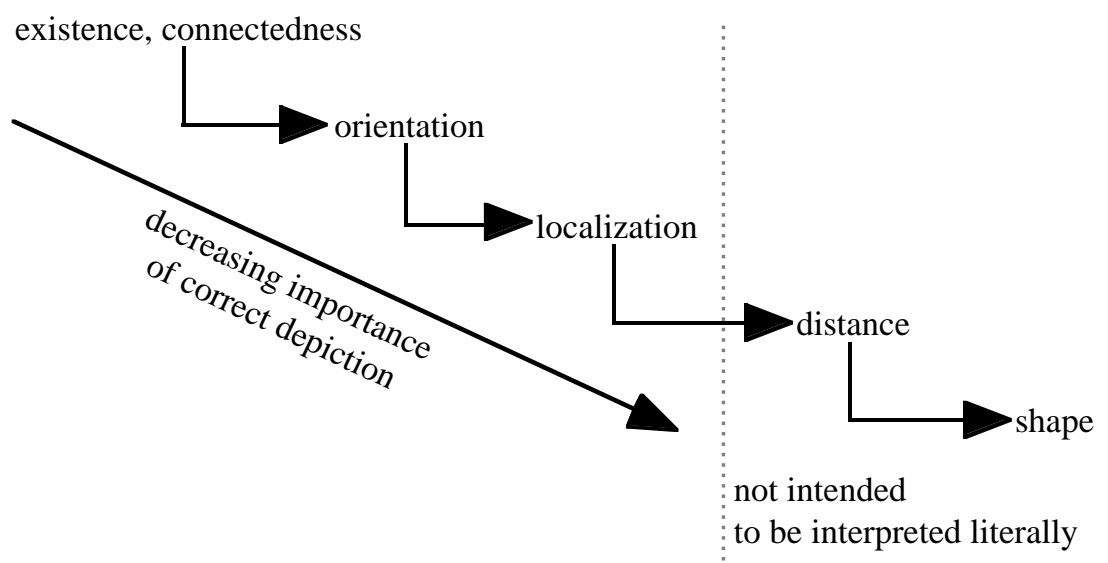

Fig. 4. Example for a depictional precedence hierarchy

\section{Conclusion and Outlook}

We have presented an approach to modeling the interpretation of geographic maps. Maps are viewed as a part of a representational system involving geographic environments on one hand and knowledge about these environments on the other. In this way, maps serve as media for communicating geographic knowledge and can be used for solving geographic problems. To interpret the contents of these media, knowledge about maps in general and knowledge about the specific type of map involved is necessary. Much of the knowledge applicable to a given level of representation can be meaningfully applied to other levels of representation as well, thus adding to the problem solving capabilities. However, the application of a given piece of knowledge may not be correct on all levels of representation. Certain kinds of cartographic misinterpretation can be explained by the application of inappropriate knowledge for a given level of generalization.

Considering the spatial constraints on the map making process, i.e. fitting spatially represented analogical knowledge together with propositionally represented symbolic knowledge into a single spatial representation medium and expecting that symbolic content, spatial locations, and spatial relationships all provide meaningful information, may appear as impossible as squaring the circle. From a theoretical 
computer science point of view a correct and complete map generation and interpretation is considered intractable. Expert cartographers as well as national mapping agencies therefore have argued that automatic map generalization - though quite desirable - is practically and economically useless [Müller et al. 1995].

As culturally trained map readers people can make good use of maps for practical problem solving; usually we do not become aware of conflicts of representation principles. Our cognitive abilities appear to be well adapted to the evolution of maps. From a cognitive science point of view, the challenge in studying map representations lies in understanding and modeling the cognitive processes that perform the map interpretation tasks. Although human solutions to map problems are not optimal in the sense of classical theoretical computer science, people easily come up with very useful solutions. It is an interesting challenge for cognitive science to find out about the solutions of human map interpretation processes.

A promising approach towards dealing with the conflicts described is to study human cognitive conflict resolution. These processes can be formally described in terms of information structures like the depictional precedence hierarchy of cartographic generalization. The formal descriptions can be implemented and explored on a computer.

More specifically, questions concerning generation, structure, and interpretation of maps like the following need to be addressed:

(1) What are the map maker's options for resolving conflicts between competing geographic features in the map making process?

(2) Which map features can transmit multiple messages and in which ways may these messages conflict or harmonize? Which role plays the context of features in a map?

(3) What kind of general map knowledge is employed in a given interpretation task and to what extent does specific knowledge carry over from one given situation to another?

The insights gained in these investigations can lead to formal models of map representation and use:

(4) How can we employ specific and general knowledge principles for goaldirected map interpretation?

(5) How can we describe contextual relationships between map components and how can these relationships represent conflicting aspects?

(6) Which knowledge structures can we employ for resolving conflicting space allocation in the knowledge mapping process?

Finally, explorative comparison between empirically and formally achieved results should be carried out:

(7) What are the most striking differences between the formal knowledge structures and the human conflict resolution strategies?

(8) To what extent are the map's capabilities to convey conflicting contents covered by formal descriptions of contextual relationships?

(9) How do formal and human map interpretation compare w.r.t. the interactions between general and specific map knowledge? How are map interpretations misled by the task the map user wants to solve? 
(10) In which ways can we take the empirically derived map interpretation biases into account in the map generation process? Can we anticipate the map user's expectations to improve the mapping results?

(11) Can formal interpretation processes profit from insights into the superior human map generation abilities and if so, in which ways?

\section{Acknowledgments}

We acknowledge stimulating discussions at the NCGIA initiative 21 specialist meeting on formal models of common-sense geographic worlds in San Marcos, Texas, November 1996, and at the 2 nd colloquium of the priority program on spatial cognition of the Deutsche Forschungsgemeinschaft in Bonn, February 1997. Special thanks are due to the members of the Hamburg spatial cognition projects.

\section{References}

Bagrow, L. (1985). History of cartography. Chicago, Ill.: Precedent Publishers.

Barkowsky, T., Freksa, C., Berendt, B., \& Kelter, S. (in press). Aspektkarten Integriert räumlich-symbolische Repräsentationsstrukturen. In C. Umbach, M. Grabski \& R. Hörnig (Eds.), Perspektive in Sprache und Raum. Wiesbaden: Deutscher Universitätsverlag.

Bertin, J. (1981). Graphics and graphic information-processing. Berlin: de Gruyter.

Eco, U. (1976). A theory of semiotics. Bloomington, Ind.: Indiana University Press.

Egenhofer, M. J., \& Mark, D. M. (1995). Naive geography. In A.U. Frank \& W. Kuhn (Eds.), Spatial information theory. A theoretical basis for GIS. LNCS 988 (pp. 1-15). Berlin: Springer.

Frank, A. U., \& Timpf, S. (1994). Multiple representations for cartographic objects in a multi-scale tree - An intelligent graphical zoom. Computers \& Graphics, 18(6), 823-829.

Freksa, C., \& Barkowsky, T. (1996). On the relation between spatial concepts and geographic objects. In P. Burrough \& A. Frank (Eds.), Geographic objects with indeterminate boundaries (pp. 109-121). London: Taylor \& Francis.

Freksa, C., \& Röhrig, R. (1993). Dimensions of qualitative spatial reasoning. In N. Piera Carreté \& M. G. Singh (Eds.), Qualitative reasoning and decision technologies, Proc. QUARDET'93, CIMNE Barcelona 1993 (pp. 483-492).

Hake, G., \& Grünreich, D. (1994). Kartographie (7. Aufl.). Berlin, New York: de Gruyter.

Head, C. G. (1991). Mapping as language or semiotic system: Review and comment. In D. M. Mark \& A. U. Frank (Eds.), Cognitive and linguistic aspects of geographic space (pp. 237-262). Dordrecht, Boston, London: Kluwer Academic Publishers.

Kanizsa, G. (1979). Organization in vision. New York: Praeger.

Kosslyn, S. M. (1994). Elements of graph design. New York: Freeman. 
Lemon, O., \& Pratt, I. (1996). Putting channels on the map: Imperfect infomation flow in a formal semantics of (geo)graphical information systems. Information theoretic approaches to logic, language, and computation. Proceedings of the 2nd Conference on Information Theoretic Approaches to Logic, Language, and Computation (ITALLC) (pp. 117-128). London Guildhall University: Department of Psychology.

MacEachren, A. M. (1995). How maps work: representation, visualization, and design. New York, London: The Guilford Press.

Monmonier, M. (1996). How to lie with maps (2nd ed.). Chicago: Univ. of Chicago Press.

Montello, D. R. (1993). Scale and multiple psychologies of space. In A. Frank \& I. Campari (Eds.), Spatial information theory: A theoretical basis for GIS. LNCS No. 716 (pp. 312-321). Berlin: Springer.

Müller, J. C., Weibel, R., Lagrange, J. P., \& Salgé, F. (1995). Generalization: State of the art and issues. In J. C. Müller, J. P. Lagrange, \& R. Weibel (Eds.), GIS and generalization: Methodology and practice (pp. 3- 17). London: Taylor \& Francis.

Palmer, S. E. (1978). Fundamental aspects of cognitive representation. In E. Rosch \& B. B. Lloyd (Eds.), Cognition and categorization (pp. 259-303). Hillsdale, NJ: Lawrence Erlbaum.

Pratt, I. (1993). Map semantics. In A. Frank \& I. Campari (Eds.), Spatial information theory: A theoretical basis for GIS (pp. 77-91). Berlin: Springer.

Schlichtmann, H. (1985). Characteristic traits of the semiotic system 'map symbolism'. The Cartographic Journal, 22, 23-30.

Schlichtmann, H. (1991). Plan information and its retrieval in map interpretation: The view from semiotics. In D. M. Mark \& A. U. Frank (Eds.), Cognitive and linguistic aspects of geographic space (pp. 263-284). Dordrecht, Boston, London: Kluwer Academic Publishers.

Wang, D. (1995). Studies on the formal semantics of pictures. ILLC Dissertation Series 1995-4. Institute for Logic, Language and Computation, Universiteit van Amsterdam.

Wood, D., \& Fels, J. (1986). Designs on signs: Myth and meaning in maps. Cartographica, 23 (3), 54-103. 